Sección central

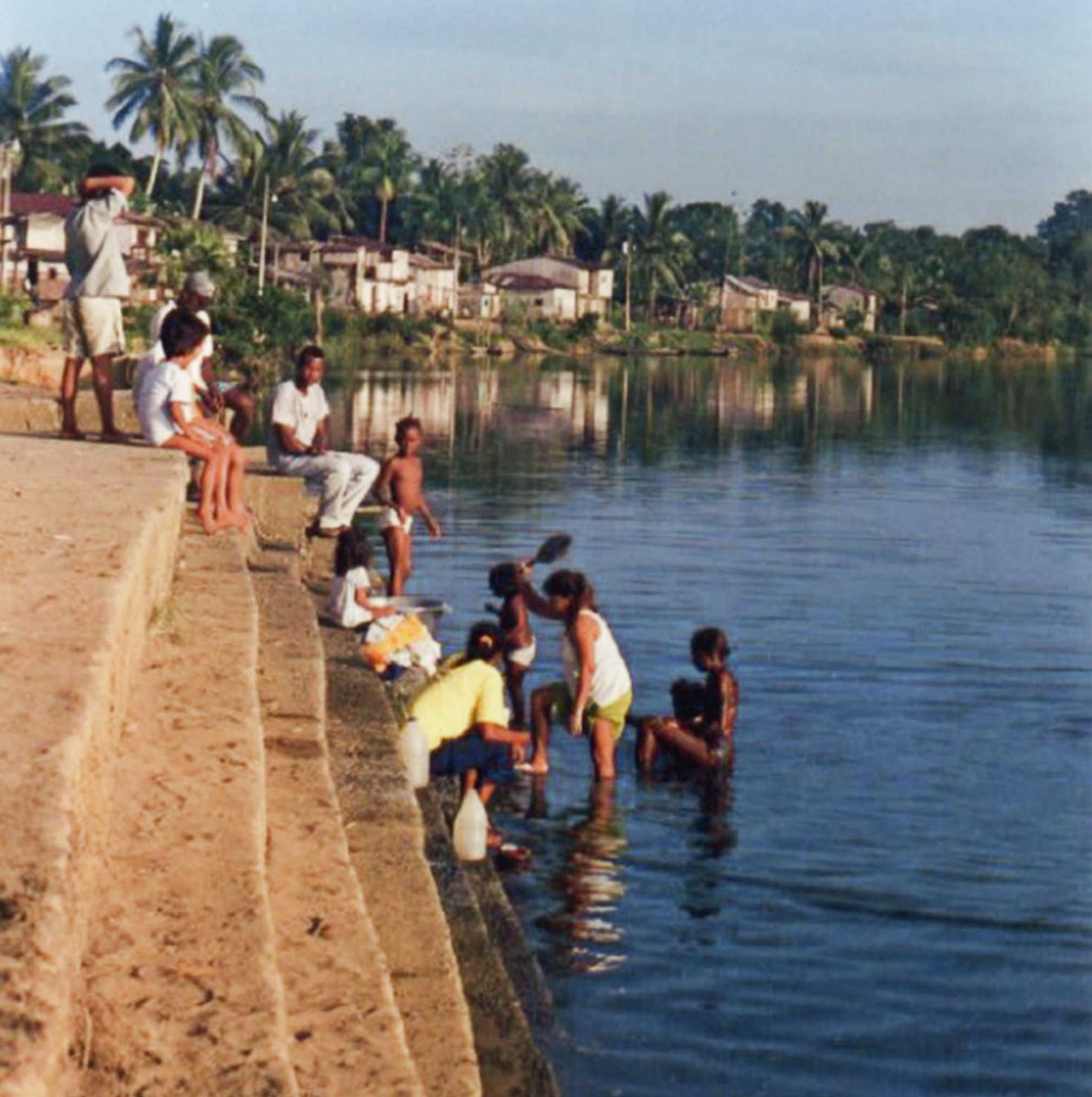




\section{Procesos de comunalización y etnización en la construcción de identidades colectivas de la gente afro del Ecuador}

\section{Artículo de Reflexión}

Recibido: 10 junio de 2017

Aprobado: 15 julio de 2017

Lucy Santacruz Benavides

Universidad Mariana, Pasto, Colombia

Isantacruzb@umariana.edu.co

Cómo citar este artículo: Santacruz Benavides, Lucy (2018). Procesos de comunalización y etnización en la construcción de identidades colectivas de la gente afro del Ecuador. Estudios Artísticos: revista de investigación creadora, 4 (4) pp.62-75. DOI: https://doi.org/10.14483/25009311.12933 Río Santiago, Maldonado (2001). Fotografía: Lucy Santacruz Benavides

\section{Resumen}

En estas páginas presento algunas reflexiones frente a los procesos de construcción de identidades colectivas de las poblaciones afro del norte del Ecuador, mostrando cómo la formación discursiva de las comunas y las etnias expresan representaciones identitarias, resultado de tensiones, negociaciones y posicionamientos políticos de las poblaciones, en su agencia colectiva frente al Estado. Las identidades, serán entonces consideradas como escenarios de lucha, donde se negocian y se intentan subvertir relaciones de poder. Para esto, inicialmente hago referencia a algunos hitos históricos que permiten ubicar el poblamiento afro de Esmeraldas como escenario de resistencia, momentos que van dibujando, a su vez, el lugar de esta zona en el ideario nacional ecuatoriano; posteriormente me refiero a la constitución de la Comuna Rio Santiago Cayapas, para mostrar las maneras particulares como se dio en este escenario lo que llamo proceso de comunalización, y finalmente retomo algunos elementos relevantes frente a la etnización como resultado adelantado por el movimiento afroecuatoriano.

\section{Palabras clave}

Afroecuatoriano, etnicidad, identidades.

Processes of Communalization and Ethnization in the Construction of Collective Identities of the Afro People of Ecuador

\section{Abstract}

In these pages I present some reflections on the construction processes of collective identities 
of the Afro populations in the north of Ecuador, showing how the discursive formation of the communes and the ethnic groups expresses representations of identity, which are a result of tensions, negotiations and political stands of the populations in their collective agency before the State. These identities will then be considered as battle scenarios, where power relations are negotiated and subverted. For this, I initially refer to some historical milestones that allow us to locate the Afrodescendant settlement of Esmeraldas as a scenario of resistance, moments that, in turn, delineate the place of this region in the Ecuadorian national ideology. Subsequently, I refer to the constitution of the Rio Santiago Cayapas Commune, to show the particular ways in which the process of communalization took place in this scenario. Finally, I take on some relevant elements surrounding ethnization as the result obtained by the Afro-Ecuadorian movement.

\section{Keywords}

Afro-Ecuadorian, ethnicity, identities.

\section{Processus de communalisation et d'ethnicisation dans la construction des identités collectives du peuple afro en Equateur}

\section{Résumé}

Dans ces pages je présente quelques réflexions sur les processus de construction des identités collectives des populations afro du nord de l'Equateur, montrant comment la formation discursive des communes et des groupes ethniques exprime des représentations identitaires, des tensions, des négociations et des positions politiques des populations dans son agence collective devant l'Etat. Les identités seront alors considérées comme des scénarios de bataille, où les relations de pouvoir sont négociées et tentées d'être subverties. Pour cela, je me réfère d'abord à quelques étapes historiques qui nous permettent de situer la colonie afro-descendante d'Esmeraldas comme un lieu de résistance. Ces moments, à leur tour, dessinent la place de cette zone dans l'idéologie nationale équatorienne. Plus tard, je me réfère à la constitution de la commune de Rio Santiago Cayapas, pour montrer comment ce scénario est apparu, ce que j'appelle le processus de communalisation, et, finalement, je ramasse quelques éléments pertinents autour de l'ethnicisation comme un résultat obtenu par le mouvement afro-équatorien.

\section{Mots clés}

Afro-équatorien, ethnicité, identités.

\section{Processos de comunalização e etnização na construção de identidades coletivas}

\section{Resumo}

Nestas páginas apresento algumas reflexões sobre os processos de construção de identidades coletivas das populações afrodescendentes do norte do Equador, mostrando como a formação discursiva das comunidades e etnias expressam representações identitárias resultado de tensões, negociações e posicionamentos políticos das populações, sem sua agência coletiva frente ao Estado. As identidades serão então consideradas como cenários de luta, de onde se negociam e se têm em vista subverter relações de poder. Para isto, inicialmente faço referência a alguns marcos históricos que permitem localizar o povoamento afrodescendente de Esmeraldas como cenário de resistência, momentos que vão desenhando, por sua vez, o lugar desta região no ideário nacional equatoriano; posteriormente me refiro à constituição da Comuna Rio Santiago Cayapas para mostrar os modos particulares como se deu, neste cenário, o que chamo de processo de comunalização e, finalmente, retomo alguns elementos relevantes frente à etnização como resultado alcançado pelo movimento afro-equatoriano.

\section{Palavras-chave}

Afro-equatoriano, etnicidade, identidades.

\section{Ruraikuna kulunisacionmanda Nukanchipa wagki ainakunamanda kai sug llagta Ecuadurmanda}

\section{Maillallachiska}

Kai pangakunapi kawachinimi allí iuiari nukanchipuramanda iana runakunamanda kai llagta Ecuadurmanda kawachispa imasami ka kai ichaikuikuna kai chasami kawachiri imasami paikuna kankuna i imasami chaskinkuna paikuna i chukuiñakui i suma rimai niskakuna maipimi kaugsankuna, 
maipi atun llagtamanda. Kai llagta kamgapami ka kawachiska imasami suma tsabajaskaikuna, Achka jumbiwa, maipimi tsukari, chasami pipas kidanga taita mandadukidaska, kaipa ka kaiarimi sug parlakuna chasami kawachimi maipi kaugsaskaku ianakuna kai llugta esmeraldas chipimi kawarii iapa tsabaskakuna, chasami, kilkaskamanda kai iuarpi kidaska suma antiura kaugsaskakunamanda atun llagta alpa suti Ecuadurmanda:chasami nikuni kai iiakuna imasami ruraska kaska atun iaku Santiago kaiapas kawachingapasugrischa imasami kawachiriska kai alpapi chasamikai ka suma tukuikuna Sugllapi kaska, ña tukuchingapa kaiarimi sug ima ministidikunawa kai Nukanchipa pura Nukapa chasami kangapa kai iana runakunapa.

\section{Rimangapa Ministidukuna}

lana runakuna Ecuadurmanda, nukanchipura, pitakaskakuna.

\section{Introducción}

Hablar de comunalización y etnización nos remite a pensar en procesos identitarios, en la relación entre los grupos subalternos y el Estado, dos procesos que emergen dentro de múltiples articulaciones y que a su vez refieren posicionamientos distintos, los cuales en ocasiones pueden ser leídos incluso como contradictorios. Las identidades no son fijas, su movilidad se corresponde con la eficacia simbólica y política con la que actúan, es en esta tensión histórica y localizada, entre el Estado como expresión de un poder gestor del proyecto moderno y los grupos subalternos, donde adquieren sentido las identidades tanto comunales como étnicas.

Entiendo la comunalización como el proceso organizativo a través del cual un colectivo se nombra comuna, en la búsqueda de reconocimiento legal de parte del Estado y frente a la construcción de un territorio autónomo; para nuestro caso particular, representa un escenario donde se tejen facultades organizativas de los pueblos afro, así como, escenarios de disputa frente a los impulsos nacionales de modernización. Por su parte la etnización marca un nuevo régimen de representación de estas identidades colectivas, donde se dibujan a su vez la conformación de los movimientos sociales y nuevos escenarios de negociación frente al Estado, en donde la cultura adquiere un lugar relevante como posicionamiento político y matriz identitaria.

La comunalización y la etnización no son procesos contrarios o complementarios, no aparece el uno cuando se termina el otro, tienen momentos de simultaneidad y distanciamiento, constituyen articulaciones distintas y tienen trayectorias específicas, sin embargo la dinámica de cambio que viven las organizaciones muestran dos momentos de reivindicación identitaria que pueden parecer divergentes. Contradicciones supuestas que causan tensión al interior de las dinámicas organizativas, por esta razón, es necesario conocer estos procesos en su complejidad particular, de tal manera que permitan articular las potencias organizativas desarrolladas en sus trayectorias y evidenciar las funcionalizaciones conseguidas por los entes gubernamentales, de escenarios originalmente concebidos como espacios de reconocimiento y agencia colectiva.

La historia de vida de la Comuna Río Santiago Cayapas ${ }^{1}$ nos permitirá etnografiar una experiencia de tal relacionamiento, donde la modernidad como proyecto hegemónico se diversifica en el encuentro o desencuentro con su exterioridad y donde los grupos subalternos construyen escenarios alternativos. En un primer momento a partir de la memoria de formación de un grupo cimarrón, donde el norte de Esmeraldas es imaginado por las élites del poder del Estado como un territorio "libre" al que es necesario controlar y explotar, un segundo momento con la compra de tierras de la comuna, como territorio colectivo donde se funda un proyecto de vida autónomo y finalmente un tercer momento en la legalización de la Comuna como tal y su inserción en un tipo de modernidad.

De manera complementaria con nuestro análisis, la historia de la comuna nos permitirá reflexionar

\footnotetext{
1 La Comuna Río Santiago Cayapas pertenece a lo que se ha denominado la región biogeográfica del Litoral Pacífico, comprendida en tres naciones: desde el sur de Panamá, pasando por todo el occidente colombiano, hasta llegar a la zona norte de Esmeraldas en el Ecuador. La provincia de Esmeraldas se encuentra ubicada al nororiente del Ecuador, limita al norte con la frontera colombiana, al occidente con el océano pacífico, al sur con la provincia de Manabí, al sur oriente con la provincia de Pichicha y al noroccidente con las provincias de Imbabura y Carchi. La Comuna Río Santiago Cayapas, se ubica al norte de la provincia de Esmeraldas.
} 
sobre los desafíos y nuevos sentidos generados por la etnización en los últimos años, particularmente a partir del surgimiento del movimiento afroecuatoriano y su propuesta organizativa de la Gran Comarca, desde un reposicionamiento de la cultura, de su significado en una lucha por el reconocimiento de derechos colectivos frente al Estado, resultado conseguido en el Ecuador en la Reforma Constitucional de 1998.

\section{Historia de un territorio "libre"}

La Comuna tiene una historia de 120 años, ocupa un extenso territorio de bosque húmedo tropical, de aproximadamente 62.000 hectáreas de tierra. En ella viven cerca de 16.500 pobladores afroecuatorianos, ${ }^{2}$ descendientes del grupo cimarrón liderado por Alonso de Illescas.

Esta historia inicia a mediados del siglo XV, época colonial, cuando grandes embarcaciones que venían del Viejo Mundo, comerciaban con gente esclavizada traída de las costas occidentales del continente africano. Para entonces se registra la llegada de una embarcación de propiedad de un sevillano que naufraga en las costas de Esmeraldas, dejando libres a 17 hombres y 6 mujeres. Liderados por Alonso de lllescas, esclavizado ladino quien adopta el nombre de su amo; los negros fugitivos se adentran en la región de Esmeraldas conformando uno de los palenques más fuertes del Litoral Pacífico (Rueda, 1999, p. 5). ${ }^{3}$

Durante todo el periodo colonial, la zona permanece relativamente aislada de los centros de poder ubicados en la sierra, considerada entonces como un territorio libre al que llegan esclavizados huidos de las minas de Barbacoas y las haciendas del Chota. Es tan solo hasta los siglos XVII y XVIII donde tienen lugar algunas expediciones en la zona, como parte de proyectos viales que tan solo fueron posibles hasta 1735, cuando se fundaron los pueblos de Limones, San Lorenzo, Concepción,

\footnotetext{
2 Información del presidente de la Comuna Río Santiago en el 2001, no existe un censo poblacional oficial.

3 La crónica de Miguel Cabello de Balboa, misionero que visitaba la región en 1577, es el primer documento escrito referenciado por los historiadores. En ella se ubica el lugar de naufragio en la ensenada de Portete y la procedencia de los esclavizados desde las costas de Guinea. (Rueda 1999).
}

Río Verde y Cayapas, incentivando la explotación de sus minas de oro. Posterior a la independencia de Esmeraldas en 1820, el Estado ecuatoriano declaró la zona como apta para la colonización de la región, permitiendo la entrada de compañías extranieras. En 1861 la Ecuador Land Company adquiere la concesión de 200.000 "cuadras de terreno". 4

Simultáneamente a estos procesos económicos se da la "manumisión de esclavos y la anulación jurídica del concertaje, que en la provincia se produce desde 1854 a 1894 en minas y haciendas de la zona norte" (Rivera, 1986, p. 27). Según Wolf (citado por Escobar 1999, p. 9), "luego de la manumisión de esclavos, negros de las haciendas de Imbabura fueron a poblar el Río Santiago, el Uimbí, el Cachabí y el Bogotá" todos estos ríos del norte de la provincia de Esmeraldas.

Es en la figura del negro concierto, donde los antiguos negros esclavizados y libres se acogen al trabajo de las minas y las haciendas contrayendo deudas impagables, que sometían la vida de familias enteras a las órdenes de un patrón. ${ }^{5}$ Las deudas adquiridas pasaban de generación en generación, lo cual puede ser visto como una expresión posterior de la esclavización.

\section{Un proyecto de vida autónomo}

Como respuesta a esta nueva dinámica esclavista, las poblaciones del Río Santiago deciden comprar su libertad junto con un extenso territorio comprendido entre el rio Santiago y el río Cayapas. Sesenta y dos mil hectáreas de tierra fueron compradas a la viuda de Viteri, propietario de las tierras por concesión de Juan José Flores, primer presidente ecuatoriano, quien otorgó a este personaje derechos sobre la explotación de las tierras, en correspondencia a su apoyo en las guerras independentistas. ${ }^{6}$

El 2 de junio de 1885, Nicolás Jiménez y Estanislao Ponce, se presentan como postores del terreno,

\footnotetext{
$4 \quad$ Al respecto ver: Fredy Rivera, 1986, p. 27.; Marta Escobar, 1999, p. 9.

5 Valdez Flavio, líder de la región, Colon Eloy, comunicación personal, agosto 31 de 2001.

6 Cososo Hermógenes, Líder Colon Eloy, comunicación personal, enero 3, 2001
} 
expresando que el remate de la tierra lo hacían "por sí y a nombre de 62 compradores".7 Es por esto, argumentan los líderes de la región, que las tierras no se pueden vender, las tierras no fueron donadas por el Estado, fueron compradas por sus antepasados como proyecto de vida autónomo. Proyecto que mantenía la propiedad colectiva de su territorio de acuerdo a las normas establecidas por su tradición, es decir, por una concepción de apropiación del espacio de acuerdo a la capacidad del trabajo, por los principios de solidaridad y redistribución dentro de las familias extensas, así como la herencia de creencias y normas de conducta frente a las relaciones con la selva que permitieron mantener los bosques y las familias dentro de una relación armónica.

Para ese tiempo las tierras de la comuna se encontraban bajo la jurisdicción de Concepción, la primera Parroquia en la zona, seguida por la Tola y Limones, que fueron centros de acopio de las haciendas y las pequeñas poblaciones asentadas en los ríos. Lugares donde comenzó a surgir el comercio de los productos de la zona hacia fuera y de alimentos y nuevos productos hacia adentro.

Entre los periodos de auge económico propiciado por compañías extranjeras se destaca el tabaco desde 1850 hasta 1890 , la tagua desde 1870 hasta 1940, y el boom bananero en 1948 hasta su caída en 1965. A partir de 1970 hasta hoy la explotación de madera constituye la fuente de recursos más fuerte de la región. (Naranjo, 1986, p. 43).

Con cada auge y con la apertura del comercio, la construcción de carreteras consigue finalmente la integración de la zona al resto del país. Poco a poco la entrada de grandes tráileres en los que se carga el bosque talado; llegan las compañías con el dinero, convierten a los jóvenes en jornaleros, movilizan pueblos enteros, marcan nuevas jerarquías.

Como cuenta Whitten (1992), las prácticas de subsistencia como la caza, la pesca y la recolección se debilitan sin desaparecer, entre un boom y otro.

A lo largo de la historia moderna de la zona norte de Esmeraldas, las presiones externas sobre la

7 Copia de Escrituras notariales, Archivo ministerio de Agricultura Quito.

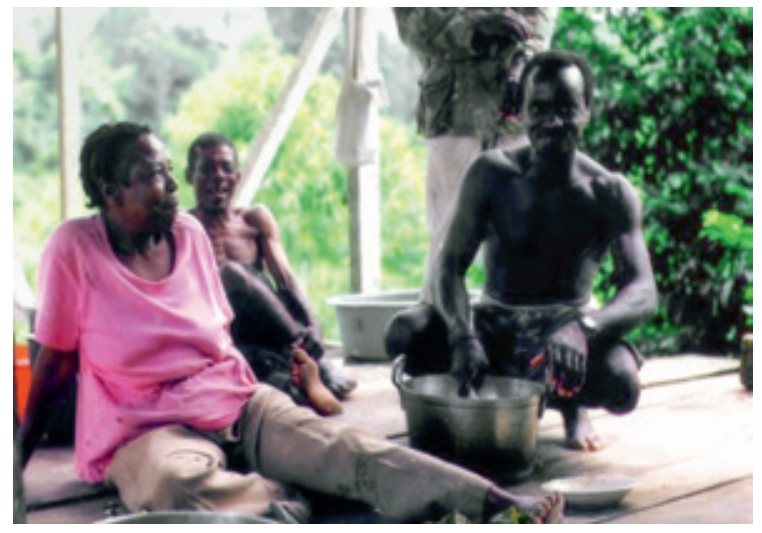

Colon Eloy (2001). Fotografía: Lucy Santacruz Benavides.

misma le han creado una atmósfera de cambio inminente. Primero fueron los negros una fuerza laboral potencial, con su exitosa usurpación de las tierras de los indígenas. Luego vinieron los explotadores que proporcionaron un mercado, aunque pequeño para los productos forestales. Empresarios extranieros provocaron los auges esporádicos necesarios para la expansión de la fuerza laboral negra, mientras que la riqueza del medio posibilitaba el trabajo de subsistencia entre un boom y el siguiente. (Whitten, 1992, p. 45).

Todo esto trae consigo transformaciones significativas en la forma de vida de la región, presiones territoriales, como nuevas dinámicas económicas hacen necesario se de una nueva legalización de las tierras dentro de la figura de Comuna.

\section{Legalización de la comuna}

Con la entrada del banano en 1948 a la provincia de Esmeraldas, inicia un nuevo proceso tanto económico como político, se insertan formas de producción capitalista, como el cultivo intensivo de la fruta, el trabajo asalariado y la búsqueda de excedente para los grandes productores, en este caso es la compañía Astral propiedad de un canadiense quien ingresa a la zona comprando e invadiendo tierras.

Según autores como Naranjo el surgimiento del Boom bananero alterará significativamente las condiciones tradicionales de vida, "la economía recolectora cede rápidamente frente a un sistema de agricultura comercial; recolectores y pescadores 
devienen en campesinos, cuando no en asalariados" (Naranjo, 1980, p. 32). El auge bananero trajo cambios fuertes en la organización social de la región, las tareas realizadas de acuerdo a la producción y comercialización del banano inserta en la zona nuevas formas de subsistencia donde el recurso monetario se convierte en movilizador social. Como lo menciona Rivera (1986, p. 35), la posesión o no del dinero marca una diferenciación de status sociales que van desde trabajadores y medianos productores, personal administrativo y de servicios, estibadores y cargadores en los muelles, hasta grupos de pobladores rurales con relaciones esporádicas a nivel de transportación.

La compañía comienza a comprar la producción, incentiva la venta de tierras, incluso invadiendo aquellas que por no tener sembríos considera como baldías. Es entonces cuando volver a las escrituras y a los argumentos que los mayores dejaron como certificado de un derecho sobre tierras colectivas se hace necesario.

La organización de la comuna hasta entonces no ha sido reconocida por el Estado, sus tierras son consideradas baldías y las escrituras como no válidas, todo esto detrás del argumento de que no existe una ocupación "real" del territorio, lo que evidencia concepciones antagónicas, pensamientos contrapuestos, entre el territorio como espacio vital y la tierra como fuente de extracción de recursos. Dejando como única posibilidad la defensa de su territorio dentro de lo que se conoce como régimen de comunas. Una manera de normalización que permite un cierto grado de autonomía territorial y de reconocimiento de un actor político como lo es la organización de la comuna.

La comuna es una forma administrativa de reconocimiento de un territorio colectivo, que el gobierno ecuatoriano había ya implementado en otros lugares, a partir de la ley de comunas. ${ }^{8} \mathrm{La}$

8 "Ley de Comunas: una comuna es una entidad reconocida jurídicamente y que, de acuerdo a la ley de Comunas de 1937, puede tener varios objetivos a) "Comunas agrarias con patrimonio común a tierras adecuadas, generalmente bajas, asi como tierras altas de pastoreo, explotación maderera y páramos". b) "Comunas de explotación en común... se aplica a los que siendo sus miembros propietarios de parcelas de tierras altas o páramos, allí se proveen de leña y tienen derecho a conservar animales..." c) "Comunas de aguas: se presentan como un
Comuna Río Santiago Cayapas, como organización campesina, se registra en el año de 1952 de acuerdo al régimen de comunas dictado por el Ministerio de Agricultura.

\section{La comunalización en el Ecuador}

La comunalización como un medio utilizado por los Estados para ejercer su soberanía, es un proceso que se institucionaliza en el Ecuador en 1937, con la promulgación de la Ley de organización y régimen de las comunas. A partir de la instauración de esta ley se inicia la legalización de territorios colectivamente ocupados en diferentes localidades, sin ninguna referencia a su historia particular, como expresa la Ley:

“Todo centro poblado (...) que existiera en la actualidad o que se estableciera en lo futuro y que fuere conocido con el nombre de (...) caserío, comunidad, parcialidad o cualquiera otra designación, llevará el nombre de Comuna." 9

La noción de comuna responde a experiencias de diferentes localidades caracterizadas por formas de vida "comunitaria", en las que se reconoce una apropiación colectiva del territorio, donde no existe la propiedad privada y donde se mantienen mecanismos de redistribución y reciprocidad. Dichas categorizaciones generales que hace el Estado permite a la ley ser aplicable a diversos casos. Como lo sostiene Hurralde (1980, p. 108). "La ley muestra una clara intención de utilizar la realidad preexistente para designar conglomerados humanos a los que se aplican sus disposiciones". Consigue establecer categorías que legitiman la existencia de organizaciones locales que permitan su control de manera diferenciada.

Con dicha normatividad se instala una forma de gobierno interno a nombre del Cabildo, conformado por seis comuneros con diferenciaciones jerárquicas. El cabildo junto con los lineamientos

grupo de propietarios indígenas o mestizos que ya por haber solicitado su adjudicación o haber realizado la captación de aguas tiene derecho a su uso y goce... Los habitantes podrán poseer bienes colectivos como tierras de labranza." (Figueroa, 1997, p. 190)

9 Ley y Reglamentos de desarrollo Agrario, marzo de 1998. Archivo Ministerio de Agricultura Quito. 
definidos por la ley que lo respalda hace parte de las transformaciones que se debieron asumir para conseguir una legislación que pueda articular y validar la economía interna con las políticas nacionales.

Entre los problemas fundamentales que el cabildo debía solucionar se encuentra la defensa a su territorio de invasiones de colonos manabitas impulsadas por el IERAC (Instituto ecuatoriano de Reforma Agraria y de Colonización) quien aún consideraba las tierras de la comuna como baldías. En una carta dirigida al Ministerio de Agricultura en 1977, el cabido hace las siguientes peticiones:

- (...) al señor Jefe Zonal del IERAC, se respete los límites de las tierras de la comuna Río Santiago.

- Pedimos ayuda para la construcción de una carretera para el desarrollo rural.

- La Comuna desea adquirir crédito para su desarrollo, por lo tanto solicitamos nos informe con qué entidad podemos operar.

- Es necesario un Destacamento Policial, para que se nos respete nuestra autoridad comunal. ${ }^{10}$

Las desavenencias con el IERAC muestran una primera contradicción con las políticas nacionales, pues este instituto en diferentes comunicaciones argumenta la posesión de un gran terreno baldío por parte de la comuna, para este la posesión de tal extensión de tierra no tiene asidero legal, afirmando por ejemplo: "Si nos atenemos a la Ley de Reforma Agraria y más leyes del país, la propiedad prescribe a los 15 años de abandono y, los terrenos no trabajados pueden ser objeto de reversión al Estado." (Comunicación del IERAC dirigida al Ministerio de Agricultura, 1987). ${ }^{11}$

Las políticas de colonización no tuvieron en cuenta la forma como la posesión del territorio es asumida por sus pobladores, pues sus economías no están basadas en el manejo de cultivos extensivos, los bosques y los ríos no son tomados como recursos de una economía extractiva capitalista. La fuente de alimentación se basa en el cultivo de plátano

\footnotetext{
10 Carta dirigida al Ministerio de Agricultura y Ganadería el 29 de septiembre de 1977. Firma el presidente, síndico y delegado del Cabildo. Archivo Ministerio de Agricultura Quito.

11 Archivo Ministerio de Agricultura, Quito.
}

a pequeña escala, cacao, yuca y otros cultivos de ciclo corto, complementados con la caza y la pesca.

Por otro lado las peticiones de construcción de la carretera y la solicitud de créditos muestran un interés respecto a los discursos de "progreso" y "desarrollo", que se manejan a nivel nacional, la captación de recursos a través de la madera abre una nueva ventana a las posibilidades de modernización. La entrada de nuevas tecnologías que permiten las explotaciones forestales de mayor alcance, así como la apertura de vías de comunicación, dan la entrada a nuevas formas de vida vistas desde afuera como propicias y que califican las suyas como atrasadas.

Así la práctica política de la Comuna se va transformando, desde el nombramiento del primer cabildo que tiene un periodo de 20 años, donde el eje central de la organización se da en la defensa del territorio como una lucha colectiva, hasta una organización que se desplaza hacia las relaciones comerciales y políticas que empiezan a tejerse con mayor fuerza con los centros de poder privados y del Estado, las relaciones de reciprocidad y redistribución cambian por una dinámica de trabajo asalariado, por el comercio y el valor monetario. La Comuna es desde entonces una organización encargada de administrar la entrada y salida de madera que paulatinamente fue adquiriendo costumbres politiqueras en el ejercicio de su poder.

Este interés de modernización impulsado por el Estado ecuatoriano, de la mano con la entrada de empresas madereras a la región, se enmarca en una perspectiva desarrollista que se ha complejizado en las últimas décadas, dando lugar a nuevos discursos, actores e intereses en la región. Desde la influencia de economías meramente extractivas, con un impacto ecológico muy fuerte, hasta la consideración del valor biodiverso de la región como parte del área biogeográfica del Litoral Pacífico (que comprende la frania del litoral desde el norte de Colombia hasta el norte del Ecuador); se genera una tensión entre políicas extractivas y conservacionistas, que ubican esta área como escenario estratégico en la perspectiva mundial, siendo considerada como una de las mayores reservas biodiversas del planeta. Esto último, sin detener la explotación de madera en los bosques esmeraldeños, ha traído definiciones como desarrollo sostenible o alternativas al 

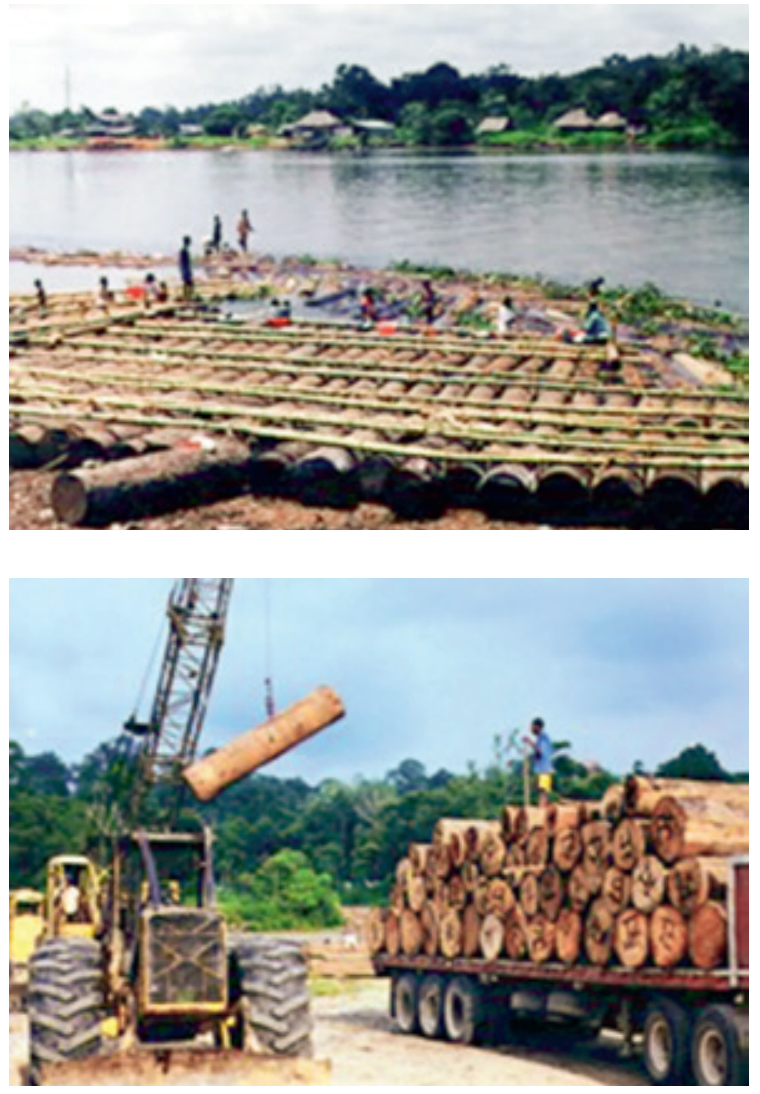

Arriba: Rio Santiago, Borbón (2001). Fotografía: Lucy Santacruz Benavides.

Abajo: Extracción de madera Timbiré (2001). Fotografía: Lucy Santacruz Benavides.

desarrollo, que son negociadas entre el Estado, las organizaciones sociales y las ONG en el marco de los intereses globales generados en la región.

Proyectos como el Prodepine, que vehiculizan recursos de "cooperación internacional", insertan en el discurso temas relacionados con el desarrollo sostenible, la biodiversidad y la cultura, que en la perspectiva de nuestro análisis cobra importancia hacia los discursos identitarios de etnización, en tanto actores institucionales que adjudican un valor dentro de sus políticas a "prácticas tradicionales" que se muestran congruentes con tales perspectivas conservacionistas. La cultura como veremos encuentra nuevos escenarios de disputa dentro de los movimientos sociales en los procesos identitarios que se han denominado de etnización.

\section{Procesos identitarios de etnización}

En este aparte, me refiero a los discursos identitarios del movimiento afroecuatoriano dentro de un conflictivo escenario de representación, en el que la cultura adquiere nuevos sentidos, en la generación de un posicionamiento político de los grupos frente al Estado. Esta tensión en los márgenes de representación identitaria es lo que he querido llamar procesos de etnización, siguiendo a Restrepo podríamos decir que la etnización se refiere al "proceso mediante el cual una o varias poblaciones son imaginadas como una comunidad étnica (... donde se) incluye la configuración de una campo discursivo y de visibilidades desde el cual se constituye el sujeto de la etnicidad" (2004, p. 272).

El concepto de etnia o grupo étnico tiene su origen en la antropología, su utilización académica como categoría de análisis responde a una cierta necesidad de clasificación de las llamadas "diferencias culturales". Los grupos étnicos fueron definidos como entidades localizables, poseedoras de características culturales tales como la lengua, el vestido, la religiosidad, entre otras. La antropología en su vertiente eurocéntrica utilizó la noción de etnia para nombrar a aquellos grupos humanos que se diferenciaban de los cánones culturales de occidente, quien a su vez clasificó tales expresiones como atrasadas en la escala evolutiva de desarrollo.

En los años 60's y 70's el concepto de lo étnico en Latinoamérica adquiere un nuevo lugar de enunciación, la participación de académicos vinculados con la antropología en una perspectiva indigenista construye en lo étnico un referente muy fuerte en los distintos grupos indígenas, buscando un tipo de puente entre las reivindicaciones identitarias, las luchas de tierras y una suerte de academia comprometida; lo que permitió a estos grupos elaborar un discurso político de reivindicación, de una serie de prácticas y conocimientos culturales, que dieron lugar al sujeto de lo étnico como sujeto político.

Para los pueblos afros las designaciones de la alteridad estaban asociadas hasta entonces en referencia a una construcción racial más que cultural (Wade, 2000 y Cunin, 2004). Este desplazamiento entre lo racial y lo cultural como referentes identitarios marca un momento de tránsito en los discursos nacionales y de instauración de lo que 
se ha llamado proceso de etnización, momento marcado por la definición política de la Carta Constitucional de 1998, en la que Ecuador se reconoce como una nación multiétnica.

La definición de lo étnico desde el concepto académico de las ciencias sociales, hasta su transformación en la construcción discursiva de la etnicidad de los movimientos indígenas y negros en el país, muestra un panorama de la etnización como proceso de construcción identitario en el que lo cultural adquiere forma y relevancia como hecho político.

En este contexto ¿qué implica hablar de una propuesta de reivindicación étnica? En el Ecuador a partir de 1998, el movimiento afroecuatoriano inicia un trabajo de reconocimiento de sus derechos como comunidad étnica, alrededor de tres aspectos fundamentales: Territorio, organización y autonomía, dentro de los cuales se menciona:

- Tener un territorio propio

- Afianzar las identidades culturales

- Conformar un poder político y representación

- Tener control sobre el manejo de recursos naturales

- La formación de una organización comunitaria ancestral

- Tener participación directa en proyectos de desarrollo

- Impulsar procesos de etnoeducación.

- (Propuesta para la Gran comarca, 2000. p.8)

La identidad dentro de los idearios de fortalecimiento de los movimientos afroecuatorianos compromete una discusión y un trabajo prolongado dentro de sus "comunidades", donde se generan las herramientas para que sus pueblos vuelvan a definir los términos de una identidad fundada en sus prácticas culturales, y desde donde se logre delinear la filosofía del movimiento mismo. Lo que contribuye, desde sus argumentos, a la superación de una crisis de valores que su condición de "discriminado" ha producido a lo largo del tiempo.

La identidad de un pueblo definido por el Estado y por la sociedad dominante, como una minoría, se la subordina y se la menosprecia, y cuando esto sucede (...) se produce en el discriminado una crisis de valores culturales, que lo llevan

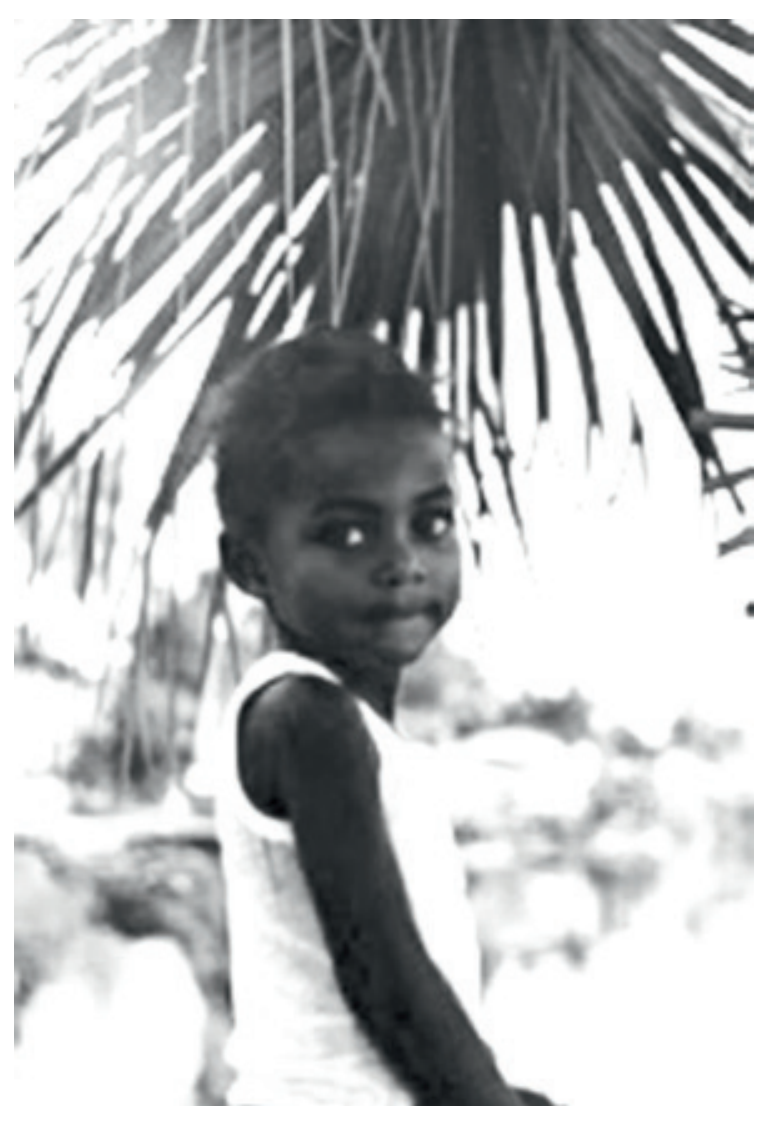

Timbiré, (2001). Fotografía: Lucy Santacruz Benavides.

a un enfrentamiento con su propia identidad. (Documento de Trabajo, Coord. García, 2000, p. 5.)

Cuando lo cultural deviene explícitamente en político, más allá de los discursos reivindicatorios, conlleva un fortalecimiento interno que nutre las posibilidades y las aspiraciones locales, lo que representa un cambio significativo en sus formas de vida. Esta dimensión de la identidad con fines políticos y de reconstrucción hacia el interior, con un sentido de posicionamiento, es lo que autores como Escobar llaman "política cultural" entendida como el "vínculo constitutivo entre cultura y política" (1999, p. 195). En este sentido el territorio es el sujeto fundamental de las luchas, de los sentidos y de los conocimientos que se tejen en los procesos organizativos, es por el territorio y en el territorio donde acontece la vida. Como menciona Escobar en el caso de Yurumangi ". A través de su nacer-crecer-conocer ejercen las muchas prácticas a través de las cuales han producido sus territorios/sus mundos desde que fueron libres." (Escobar, 2016, 
p. 16), el territorio significa autonomía, posibilidad de ser, libertad.

Los movimientos sociales afroecuatorianos proponen un encuentro hacia adentro, que permita proyecciones futuras, utilizando herramientas que articulen la vida desde los lineamientos culturales propios, llegando a formular alternativas a la modernidad, en tanto que establecen un enfoque desde donde se cuestiona conceptos como desarrollo, y se revalora proyecciones como la biodiversidad.

Sin embargo cabe preguntarse ¿por qué lo étnico se vuelve relevante?, ¿no es una manera discursiva del Estado por normar las diferencias culturales?, ¿no genera esto nuevos esencialismos como los buscados con la institucionalización de las comunas?; si constituyen maneras necesarias para lograr un reconocimiento hacia afuera y un fortalecimiento de lo propio hacia adentro, tales como lo que se ha denominado esencialismos estratégicos, ¿están los movimientos sociales conscientes de la dinámica de este concepto?-

Tanto la comunalización como la etnización expresan procesos de relaciones de poder impuestas por el Estado a los grupos locales, grupos que al no pertenecer al conjunto de la sociedad mestiza dominante, en los Estados Latinoamericanos, han sufrido de un distanciamiento que al mismo tiempo ha permitido categorizaciones y procesos de organización locales distinto respecto a la sociedad nacional, lo que algunos autores Ilamarían pensamiento fronterizo.

La comunalización constituye un primer momento de integración/articulación de los grupos dentro de las políticas del Estado, políticas que reflejan el interés de dominación de este, respecto a grupos locales dispersos que en algún sentido mantienen tensiones frente a tal poder desde su constitución interna. La noción de comunidad no es inocente en las políticas del Estado, como tampoco lo es la noción de etnia, y por lo tanto no pueden ser vistas como reflejo de características objetivas, su presencia es resultado de relaciones tensas.

El concepto de comunidad, de comuna en el Ecuador, permitió al Estado decretar políticas administrativas que agruparon y redujeron dichos grupos diferenciados dentro de políticas homogeneizadoras que buscaban en ultimas los ideales de construcción de nación. Un proyecto que le apuesta al mestizaje como mecanismo de superación de las 'inapropiadas' diferencias 'étnicas' o 'raciales' en busca de un "blanqueamiento cultural". Lo cual produjo tensiones en las localidades donde se ubicaban tales diferencias, generando procesos de exclusión y al mismo tiempo de fortalecimiento de fronteras y alianzas internas. Como los señala Whitten (1980, citado por Wade, 2000, p. 101):

Existe un nacionalismo ecuatoriano [que] se basa en una 'ideología de homogeneización étnica' o de 'mezcla racial'... Esta ideología puede utilizarse para excluir a quienes se consideran no mezclados, máxime porque la ideología tiene una 'cláusula clasificadora tácita que levanta el precio de admisión [a la nación mezclada] desde la simple mezcla fenotípica al blanqueamiento cultural (en cuanto a volverse más urbano, cristiano y civilizado y menos rural, negro e indígena).

El concepto de "blanqueamiento cultural" que nos ofrece Whitten, es clave para entender las particulares formas de relación que los referentes étnicos y raciales representan en las políticas identitarias buscadas por la élite de la época. Casi sinónimos tales referentes identitarios encaminan una política colonial hacia la transformación en un ideal fenotípico (blanqueamiento) y de comportamiento (cultural) donde los problemas raciales que dirigen su mirada hacia lo negro, como lo étnico con lo indígena, expresan una diferencia desvalorizada, discriminatoria, como rasgo casi genético ubicado en las escalas inferiores de evolución.

Siguiendo dichos ideales de nación-Estado moderno se implementaron proyectos de educación, salud, comercio, entre otros, a nivel nacional, en los que la comuna era el escenario que se ajustaba a los requerimientos de las nuevas políticas del Estado, en tanto agrupaba en "organizaciones campesinas" los dispersos grupos locales ubicados en 'la periferia': “los ecuatorianos mezclados se ven a sí mismos como verdaderos nacionales, consideran que el progreso consiste en transformar las periferias de la nación mediante el desarrollo que cambia tanto la tierra como su gente" (Whitten, 1980, citado por Wade, 2000, p102). Las periferias eran precisamente las zonas rurales donde el Estado tenía interés de implementar economías extractivas de alto alcance, con la utilización de grandes maquinarias en busca de excedentes que le permitieran 
ingresar en el comercio internacional con la exportación de materias primas, proceso que inicio y que aun esta imbricado dentro de las ideologías desarrollistas que se expandieron como ideal casi natural dentro de los proyectos de vida de las poblaciones locales y de los proyectos nacionales.

El desarrollo fue visto como una política totalizadora donde no era necesario reconocer las particularidades locales que en su relación tensa constituían más bien un problema que era necesario arrasar, se desconocían sus "historias múltiples [en favor de]... la lógica del poder colonial... esto es, desarticular los conjuntos políticos mayores a fin de atomizar al campesinado en unidades locales, autónomas y aisladas" (Saignes, 1991, p. 128). Características esenciales que simulan la codificación del 'campesinado' para imaginar su fácil manipulación.

La figura de comuna integra a los grupos indígenas y negros geográficamente ubicados como campesinos de zonas rurales, organizados dentro de un territorio colectivo, logrando articular la productividad, como la organización política de dichas localidades con los intereses nacionales.

En este sentido, las comunas son "organizaciones que fueron definidas de forma corporativa por el Estado. Sus bases fueron la supuesta o real vocación tradicional indígena hacia la producción, distribución y redistribución colectiva, y actualmente constituyen la base sobre la que se negocian y renegocian las identidades étnicas en el Ecuador." (Figueroa, 1996, p. 190).

Las etnización, por su parte, buscan cambiar los lugares de enunciación de las identidades dando prelación a los contenidos culturales, como artífices constructores de nuevas dimensiones en las relaciones de poder con el Estado y dichos grupos locales, haciendo énfasis en la cultura de cada pueblo, en las historias de vida de cada localidad, sin dejar de constituir alianzas dentro de referentes identitarios étnicos que permitan una posicionalidad y la construcción de un nuevo actor político.

Sin embargo, cuando una comunidad étnica es definida dentro de los parámetros intelectuales y políticos que constituyen lo étnico, las diferencias culturales consideradas de manera relevante en tales discursos son naturalizadas, generando tensiones frente a un deber ser de las comunidades, impulsado por los mismos movimientos sociales. Es así como en el caso de la comuna, la formación de palenques propuesta por el movimiento afroecuatoriano como nueva posibilidad organizativa, no logra adquirir sentido para sus líderes, por un lado porque desconoce la trayectoria organizativa de la comuna dividiendo el territorio de esta y por otro lado en referencia a una categoría como la de palenque que si bien hace parte importante de la historia de resistencia de los pueblos afro no tiene un referente en la memoria de los mayores que permitan leer el palenque como tal.

En Colombia, Restrepo (2004), haciendo alusión al PCN (Proceso de Comunidades Negras), movimiento afrocolombiano, introduce la categoría de Pedagogía de la Alteridad como iniciativa del proceso organizativo para difundir en sus comunidades la apropiación de una serie tanto de categorías, como de referentes históricos, importantes en tales discursos identitarios étnicos. Lo que puede aparecer como una nueva reinvención de lo propio desde esencialismos que pueden considerarse estratégicos de acuerdo al momento político de los movimientos sociales frente al Estado, pero que por otro lado pueden representar una presión desde dichos movimientos hacia sus comunidades desconociendo los procesos y proyecciones actuales de las poblaciones, llegando a caer lo que autores como Cunin (2004, p. 68) Ilaman neoracismos culturales, haciendo referencia a población afrocolombiana urbana que no encaja dentro de los discursos étnicos. ${ }^{12}$

Los desafíos en este sentido son cada vez mayores, los movimientos sociales en su negociación con el Estado, corren el peligro de normatizar lo que en algún momento es propicio en el fortalecimiento interno, en la búsqueda de sentidos, los esencialismos presentes en los procesos de etnización pueden representar el anquilosamiento de propuestas alternativas y la generación de conflicto dentro de las mismas comunidades. La cultura en este escenario pasa de ser un elemento fundante de las filosofías propias de los movimientos a un instrumento

\footnotetext{
12 Este neoracismo cultural, se basa en una valoración positiva de las diferencias y en la presentación de la alteridad como algo invariable.
} 
de negociación, es incluso mercantilizada en la gestión de recursos perdiendo los sentidos profundos en los que inicialmente es valorada por los movimientos.

\section{Referencias}

Cunin, E. (2004). Formas de Construcción y Gestión de la Alteridad. Reflexiones sobre "Raza" y "Etnicidad". En: Estudios Afrocolombianos Aportes para un Estado del Arte. Memorias del Primer Coloquio Nacional de Estudios Afrocolombianos. Universidad del Cauca.

Escobar, A. (1999). El final del Salvaje. Naturaleza, Cultura y Política en la antropología contemporánea. Bogotá: ICAHN y CEREC.

(Enero-abril 2016). Sentipensar con la tierra: Luchas Territoriales y la Dimensión Ontológica de las Epistemologías del Sur. Revista de Antropología Iberoamericana 11(1), p. 11-32. https://doi.org/10.11156/aibr.110102

https://doi.org/10.11156/aibr.110102e

Escobar, M. (1990). La Frontera Imprecisa. Lo Natural y lo Sagrado en el Norte de Esmeraldas. Quito: Centro de Cultura Afroecuatoriano.

PMCid:PMC488062

Figueroa, J. A. (1996-1997). Comunidades Indígenas: Artefactos de Construcción de las Identidades Étnicas en los conflictos Políticos del Ecuador Contemporáneo. Revista colombiana de Antropología V (XXXIII), p. 196.

Garcia, J. (2000). Sobre la Gran Comarca de Indios y negros en Ia Provincia de Esmeraldas. Quito. Documento de Trabajo.

Hurralde, D. (1980). Campesinos y Comunas. Ecuador: Colección Pendoneros. Instituto Otavale-o de Antropología.

Naranio, M. (1980). Etnicidad, Estructura Social y Poder en Manta. Occidente Ecuatoriano. Ecuador: Colección Pendoneros. Instituto Otavale-o de Antropología.

Quijano, A. (2002) iQué tal Raza! Revista Venezolana de Economía y Ciencias Sociales 6(1), p. 123- 145.

Restrepo, E. (Julio 2007). Identidades: Planteamientos Teóricos y sugerencias Metodológicas para su estudio. Revista Jangwa Pana (5).

Restrepo, E., y Rojas, A. (2004). Biopolítica de la Alteridad. Dilemas de la etnización de las colombianas negras. Conflicto e invisibilidad. Retos en los estudios de la gente negra en Colombia. Popayán: Editorial Universidad del Cauca.
Rivera, F. (1986). Campesinado y Organización en Esmeraldas. Quito:

Centro Andino de Acción Popular. CAAP.

Rueda, R. (1999). La Cultura Afroecuatoriana. Quito: Documento sin publicar.

Saignes, T. (1991). Lobos y Ovejas. Formación y Desarrollo de Ios Pueblos y comunidades en el Sur Andino (s.XVI-XX). Quito: Reproducción y Transformación de las Sociedades Andinas. Tomo 1.

Wade, P. (2000). Raza y Etnicidad en Latinoamérica. Quito: Ediciones Abya-Yala

Wallerstein, I. (1996). Abrir las Ciencias Sociales. Informe de la Comisión Gulberkian para la reestructuración de las ciencias sociales. México: Siglo XXI editores.

Whitten, N. (1992). Pioneros Negros. La cultura Afro'

Latinoamericana del Ecuador y de Colombia. Quito: Centro

Cultural Afroecuatoriano. PMCid:PMC195819

\section{Otras fuentes}

Archivo Ministerio de Agricultura, Actas de constitución, reglamentación y correspondencia de la Comuna Río Santiago Cayapas. Quito 2001. 